\title{
THE APRIL MEETING IN NEW YORK
}

The two hundred sixtieth regular meeting of the Society was held at Columbia University on Friday and Saturday, April 6 and 7, 1928. The attendance included several distinguished guests (Professors Paul Alexandroff, Moscow; C. Carathéodory, Munich; and Heinz Hopf, Berlin), as well as the following one hundred twenty-four members of the Society:

C. R. Adams, R. B. Adams, Alexander, R. G. Archibald, J. W. Arnold, A. A. Bennett, Benton, William James Berry, William Johnston Berry, Blumberg, Blumenthal, Bowden, B. H. Camp, W. B. Campbell, Caris, Carrié, E. T. Carroll, Cheney, Chittenden, Alonzo Church, Coble, Abraham Cohen, L. W. Cohen, L. P. Copeland, Currier, Curry, Dantzig, Derby, Dorweiler, Dresden, Esty, Fiske, Fite, Fithian, D. A. Flanders, Frink, Gehman, Gill, Gronwall, Hall, Robert Henderson, Hickson, L. S, Hill, Hille, Himwich, Hofmann, Hollcroft, Hotelling, Huber, Jeffery. Joffe, M. I. Johnson, R. A. Johnson, Johnston, Kasner, Keller, Kline, Knebelman, Mark Kormes, Langman, Lefschetz, Lepeshkin, Littauer, Lubin, MacColl, MacInnes, H. A. Merrill, Merriman, H. H. Mitchell, T. W. Moore, Richard Morris, D. S. Morse, Marston Morse, Moyle, Murnaghan, F. H. Murray, Musselman, Neelley, C. A. Nelson, Olds, Ore, F. W. Owens, H. B. Owens, Pepper, Pfeiffer, Phalen, Pixley, Poritsky, Post, R. G. Putnam, Raudenbush, Reddick, C. N. Reynolds, R. G. D. Richardson, Ritt, Roos, Rutt, Seely, Serghiesco, Sheffer, Siceloff, Simons, Singer, Slotnick, Smail, C. E. Smith, P. A. Smith, W. M. Smith, Sosnow, Swingle, Tamarkin, J. M. Thomas, T. Y. Thomas, Veblen, Weisner, H. S. White, W. M. Whyburn, Wiener, R. L. Wilder, W. A. Wilson, Mabel M. Young, Zariski, Zippin.

On recommendation of the Council, the Society adopted amendments to the By-Laws by which the editorial committee of the Colloquium Publications become members of the Council of the Society, on the same basis as the editorial committees of the Bulletin and the Transactions, and as the Society's representatives on the editorial committee of the American Journal of Mathematics.

The afternoon sessions on Friday and Saturday were devoted to a Symposium on Analysis Situs; they were presided over by Ex-President Oswald Veblen, relieved by Professor H. H. Mitchell at the opening of the Friday session. 
The following papers were presented: Friday, April 6: Threedimensional manifolds; generalized Riemann surfaces and knots, by Professor J. W. Alexander; The critical points of functions and the calculus of variations in the large, by Professor Marston Morse; Applications of analysis situs to algebraic surfaces, and the classification of curves on surfaces, by Professor Solomon Lefschetz; Saturday, April 7: Abstract sets from the point of view of analysis situs, by Professor E. W. Chittenden; The notion of curve and the problems that it presents, by Professor J. R. Kline; Application of analysis situs to the general theory of topological spaces, by Professor P. S. Alexandroff. The discussion was opened on Friday by Professor Heinz Hopf, and on Saturday by Professor H. M. Gehman; on both days it was carried on for a considerable time and in a lively manner by several participants, among them Professor Carathéodory. The success of this symposium fully justifies the belief that there is a gain in devoting a part of each meeting to a more concentrated field of interest than can be supplied by the usual programs of short papers.

The Friday and Saturday morning sessions were sectional: Friday morning, Section A, Analysis, Professor A. A. Bennett presiding; and Section B, Geometry, Professors T. R. Hollcroft and F. W. Owens presiding; Saturday morning, Section A, Analysis Situs and Point Sets, Professor J. R. Kline presiding, and Section B, Algebra and Applied Mathematics, Professor A. B. Coble presiding. Titles and abstracts of the papers read at the sectional sessions follow below. The papers numbered 1 to 11 were read before the Section of Analysis; Nos. 12-19 before the Section of Geometry; Nos. 20-34 before the Section of Analysis Situs and Point Sets; Nos. 35-44 before the Section of Algebra and Applied Mathematics. The papers of Adams (second paper), Ayres, Blumberg (second paper), Garver, Graustein, Gronwall, Hurwitz, Miller, Moore (second paper), Stetson, Swingle, and Whyburn were read by title. Mr. Kaplan was introduced by Professor F. D. Murnaghan, and Mr. Miller by Professor R. L. Wilder. 
1. Mr. A. O. Hickson: An application of the calculus of variations to boundary value problems.

This paper considers the boundary value problem which arises when one attempts to minimize the second variation $I_{2}(\eta)$ of the problem of the calculus of variations for a space of $n+1$ dimensions. A certain succession of classes of admissible arcs is chosen and the problems of minimizing $I_{2}(\eta)$ in these classes are considered. For each minimizing problem there is obtained the Euler-Lagrange system of ordinary linear differential equations of the second order containing a parameter $\lambda$ as a multiplier. In each class of arcs $I_{2}(\eta)$ has a minimum value, and these values form an increasing sequence $\lambda_{0}, \lambda_{1}, \cdots, \lambda_{i}, \cdots$. Also in each class there exists a set of linearly independent arcs, which give $I_{2}(\eta)$ its minimum value in the class considered, and which satisfy the corresponding boundary value problem with the parameter $\lambda$ equal to this minimum of $I_{2}(\eta)$. The results are obtained by means of well known theorems from the calculus of variations and the theory of ordinary linear differential equations.

2. Dr. G. M. Merriman (National Research Fellow): On the expansion of functions in terms of orthogonal polynomials. Preliminary report.

It is shown here, as an initial result in the field indicated in the title, that a function $f(x, y)$, harmonic in the interior of a closed, rectifiable Jordan curve and continuous in the closed region consisting of the curve and its interior, can be expanded in terms of a set of harmonic polynomials, normalized and orthogonalized with respect to the curve, the expansion in series being uniformly convergent interior to the curve. A set of such polynomials, which depend only on the curve, is exhibited.

3. Dr. C. F. Roos (National Research Fellow): Solutions of limited variation of Volterra integral equations of the first kind.

Volterra has found a continuous solution of the Volterra integral equation of the first kind, $v(x)=\int_{a}^{x} K(x, s) u(s) d s$, by reducing the equation to one of the second kind by a differentiation. W. H. Young has extended this theory to include Lebesgue integrals and has obtained a bounded solution. Both Volterra and Young have employed a differentiation to reduce the equation to a Volterra equation of the second kind. In the present paper the author shows that an integration by parts can be used to reduce the equation to the type $v(x)=u(x) \int_{a}^{x} K(x, t) d t-\int_{a}^{x}\left[\int_{a}^{x} K(x, t) d t\right] d u(s)$; this integration by parts only requires that the kernel $K(x, s)$ be integrable in $s$ and of limited variation in $x$ for all $s$ and that $u(s)$ be of limited variation. Under these hypotheses and the added hypothesis that $v(x)$ be of limited variation, it is possible to obtain a solution of limited variation in the form resulting from the integration by parts. 


\section{Mr. H. H. Pixley: Discontinuous solutions in the pro-} blem of depreciation and replacement.

In a recent paper on the calculus of variations, Dr. C. F. Roos has treated a Lagrange problem with variable end points and discontinuous integrand to enable him to develop his new dynamical theory of depreciation and replacement. He assumes that the price-time and rate of production-time curves are continuous at the time of replacement. In an actual problem this would probably not be true. In the present paper a general theory corresponding to that of Roos without the assumption of continuity at the time of replacement is developed. In particular a special problem is discussed in which the second machine is started at a time and rate different from those at which the first machine stops.

5. Professor C. R. Adams: On the linear ordinary $q$-difference equation.

The equation considered is (1): $\sum_{i=0}^{n} a_{i}(x) f\left(q^{n-i} x\right)$, in which the known functions $a_{i}(x)$ are analytic or have poles at $x=0, q$ is a constant $\neq 0$, and $f(x)$ is to be determined. Without loss of generality we may assume $a_{i}(x)=a_{i 0}+a_{i 1} x+\cdots$ (for $\left.|x|<R\right)$, with at least one of the $a_{i 0}$ not zero. These $a_{i 0}$ are the coefficients of the characteristic equations of (1). We exhibit formal power series satisfying (1) in all possible cases. When the roots of the characteristic equation are finite and not zero, and $|q| \neq 1$, we prove directly that these solutions have positive radii of convergence; their existence and convergence can be inferred from the functional equations derived by Grévy (Paris thesis, 1894; also Annales de l'Ecole Normale, (3), vol. 11 (1894), pp. 249-323), but the present treatment possesses advantages of directness, simplicity, and brevity. When the characteristic equation has infinite or zero roots or both and $|q| \neq 1$, some (never all) of the formal series may converge only for $x=0$. At $x=\infty$ the situation is similar if the $a_{i}(x)$ are analytic or have poles there. The non-homogeneous equation is also treated, with similar results.

6. Professor C. R. Adams: Note on factorial series in two variables.

This paper appears in full in the present issue of this Bulletin.

\section{Dr. Stefan Serghiesco: On a certain double integral.}

In an earlier paper, the author has introduced a new general formula giving the number of roots of a system of equations which contains differential invariants and is represented, in the case of two equations, by a difference of two integrals, a simple and a double integral. An abstract of this paper has appeared in the Comptes Rendus for January 23, 1928. In the present paper the author makes a further study of this problem and introduces a double integral which by itself gives the number of roots of the system. This integral is then extended to $n$ equations. The treatment, through differential invariants, of this problem is thus widely generalized for the case of simple common roots of a system of equations. 
8. Professors Einar Hille and J. D. Tamarkin: Contributions to the theory of integral equations. Preliminary communication.

Assuming the kernel to be integrable together with its square, the authors apply the theory of linear equations in infinitely many unknowns to a study of the characteristic values of a linear integral equation. These values are obtained as the zeros of an infinite determinant which is frequently easier to handle than the (modified) Fredholm determinant. Estimates are obtained for the rate of growth of the determinant and of the characteristic values in the general case as well as in various special cases, e.g., when the kernel is analytic in one or both variables or possesses a certain number of partial derivatives or satisfies a Lipschitz condition, etc. Extensive use is made of the theory of trigonometric and polynomial approximation in two dimensions.

\section{Professor Harold Hotelling: Stability in competition.}

Cournot's solution of the problem of duopoly was criticized by $\mathrm{J}$. Bertrand and F. Y. Edgeworth on the ground that his "equilibrium" is unstable. It does not seen to have been recognized, however, by any writer on the subject that this instability is due to the tacit assumption that the competitors are equally accessible and their products are of equal utility, not merely to the market as a whole but to every consumer. Discarding the last condition, which is artificial, a simple example typical of actual conditions is set up and its stability is demonstrated. By means of the same example there become evident a number of qualitative properties of competitive situations generally overlooked. Among these are are some which have a bearing on the "cement trust" controversy.

\section{Professor Norbert Wiener: Coherency matrices.}

The theory of harmonic analysis already developed by the author is extended to a set of several simultaneous functions, and yields an Hermitian function of the frequency, the coherency matrix. The problem, for example, of characterizing light with respect to its state of polarization may be reduced to that of transforming a "coherency matrix" to diagonal form by a Weyl "Unitärtransformation." The theory has important applications to quantum theory and the Heisenberg matrices.

\section{Dr. T. H. Gronwall: On the convergence region of $a$ power series in several variables.}

Fabry has shown that the region of convergence of a power series in $x$ and $y$ becomes a convex region when $\log |x|$ and $\log |x|$ are used as coordinates, and that this necessary condition on a convergence region is also sufficient. Hartogs and Faber have given simplified proofs, and the latter has extended the theorem to $n$ variables. The present note gives a proof (for $n$ variables) which is considerably simpler than the previous ones. 
12. Professor J. H. Neelley: Concerning covariants of th rational plane quartic curve with compound singularities. First paper.

This paper discusses covariant forms of the rational plane quartic curve with ramphoid cusp. Some covariant curves degenerate, and groups have a common characteristic. The curve $R_{2}{ }^{4}$ is taken parametrically as well as in ternary form. Some forms, which vanish identically for $R_{2}{ }^{4}$ with this compound singularity and with other singularities, are considered. Some facts previously derived from invariants are verified by covariants.

13. Professor T. R. Hollcroft: The postulation of multiple curves and surfaces on varieties in $i$ dimensions.

Numerical formulas are obtained for the postulation of a multiple line and of a multiple plane on a variety of given order in $i$ dimensions. That for a multiple line is a generalization of Noether's formula for the postulation of a multiple line on a surface. There is also found the reduction in postulation due to an intersection of two multiple lines (planes) when both lines (planes) are of the same or different multiplicities on a variety of given order in $i$ dimensions. Numerical formulas for the postulation of any given curve or surface of given multiplicity on a variety of given order in $i$ dimensions are derived from the above. The postulation of any given one- or two-dimensional manifold of given multiplicity on a variety of given order is similarly obtained.

14. Professor H. R. Phalen: Metric properties of the quadric of Moutard.

Under the assumption that a certain surface $S$ is non-developable. this paper defines a quadric of Moutard, derives its equation, and obtains the discriminant and the coefficients of the discriminating cubic, the coordinates of the center, and the mean and total curvature of $S$ and of the Moutard quadric at the origin, and enunciates the following theorems: (a) At a given point $P$ upon $S$ the Moutard quadric and the surface $S$ have the same total curvature, the same mean curvature, and the same surface normal. (b) The center of every Moutard quadric at a point $P$ upon $S$ lies outside the tangent plane to $S$ at $P$. (c) At a point $P$ upon $S$, twelve tangents may be drawn such that the corresponding Moutard quadric is non-central. (d) If the surface $S$ be defined by an equation of the form $z=f(x, y)$ in which no term of the right member is of degree less than the third, the Moutard quadric degenerates into the tangent plane counted twice. (e) If in the equation of the Moutard quadric the term in $z^{2}$ is absent, the quadric is central. In conclusion, criteria are deduced for classifying the various quadrics.

15. Professor Edward Kasner: Characterization of geodesic families.

It is shown that just as natural families may be characterized by integrability with reference to orthogonality (the author's converse of 
the Thomson-Tait theorem, 1909), so geodesic families of curves may be characterized geometrically by integrability with reference to any polarity (the base cone of the polarity may vary arbitrarily from point to point). Polar transversalities are the only projective correspondences which can serve as transversality relations (see these Transactions, July, 1928). The results are valid in space of three or more dimensions.

16. Professor Edward Kasner: The second derivative of a polygenic function.

The second derivative $\sigma=d^{2} w / d z^{2}$ of an arbitrary polygenic function $w=\phi(x, y)+i \psi(x, y)$ depends not only on the point $z=x+i y$ but also on the slope and curvature of the path of approach, that is, $\sigma=\Omega\left(x, y, y^{\prime}, y^{\prime \prime}\right)$. For a given $y^{\prime}$ the locus of $\sigma$ in its complex plane is a straight line. As $y^{\prime}$ varies this line envelopes a cardioid. If $y^{\prime \prime}=0$ (rectilinear second derivative) the locus of $\sigma$ is a limaçon. For a complete representation it is necessary to parametrize these loci (clocked cardioid, analogous to clocked circle, see Science, December 16, 1927). Higher derivatives are also discussed. This paper has been offered to the Transactions.

17. Professor W. C. Graustein: On the average number of sides of polygons of a net.

The leading theorem in this paper, stated in its simplest form, reads as follows: If in a net of regular hexagons covering the plane a finite simply connected set of hexagons is removed and the region thus vacated is covered by polygons in such a way that the number of sides issuing from each vertex in the net remains three, then the average number of sides, per polygon, of the polygons in the net remains six. Similar theorems hold for nets of squares and equilateral triangles.

18. Professor W. C. Graustein: Note on a certain type of parabola.

The author discusses certain striking properties of a parabola which is tangent to the line at infinity in a circular point.

\section{Dr. J. M. Stetson: Theorems of permutability.}

It seems to have escaped notice that a part of the classic theory of Levy transformations may be restated thus: the transformations of Laplace and Levy and the inverse of the transformations of Levy are permutable. From this follows the fundamental theorem: Any type of transformation made by combining these three is permutable, and is permutable with any other such type. This general theorem appears to include as special cases all known theorems regarding permutability of transformations of conjugate nets in which no restrictions are made as to the character of the nets, if we note that either radial transformations, or parallel transformations, but not both, may be added to the list of transformations given in the theorem. We obtain a simple proof of the permutability of transformations $F$, and of the transformations giving derived nets (and their inverses), a fact which seems to be new except for the special case of reciprocal derived nets. 
20. Professor R. L. Wilder: On connected and regular point sets.

This paper will appear in full in an early issue of this Bulletin.

21. Professor R. L. Wilder: Concerning irreducibly connected sets and irreducible regular connexes.

If the point set $M$ be irreducibly connected about a set $K$ but not about any proper subset of $K, K$ is called a basic set about which $M$ is irreducibly connected. Among the results obtained are the following: (1) If $K$ is a basic set about which $M$ is irreducibly connected, then (a) $K$ is identical with the set of non-cut points of $M$ and (b) if $K$ consists of $n$ points, the number of cut points of $M$ of order greater than one is at most $n-2$. (A point $P$ is called a cut point of order $n$ if $M-P$ is the sum of $n$ mutually separated sets.) Furthermore, if $P$ is a point of $M$ such that $M-P$ is the sum of $n$ components $M(i=1,2, \cdots, n)$, and if $M_{i} \times K=K_{i}$, then the set $K_{i}+P$ is a basic set about which $M_{i}+P$ is irreducibly connected. (2) If $M$ is closed and bounded, then (a) $M$ contains a basic set about which it is irreducibly connected; (b) $M$ contains at least two non-cut points. Special attention is paid to the case where $K$ consists of a finite number of points as well as to the case where $M$ is regular.

22. Professor R. L. Wilder: Concerning the relation of sets $F_{\text {oe }}$ to their complements in euclidean space of $n$ dimensions.

We denote by $F_{\sigma \epsilon}$ a set $M$ which is the sum of a denumerable collection of mutually exclusive, closed and bounded sets $M_{1}, M_{2}, \cdots$, whose diameters converge to zero as $n$ tends to infinity. Among the results obtained are the following: (1) If $D$ is a connected domain in euclidean space $E_{n}$ such that no set $M_{n}$ contains points of both $D$ and $E_{n}-D$, and if no set $M_{n}$ separates $E_{n}$, then $D-M \times D$ is a properly non-vacuous, arcwise connected point set. (2) Let $A$ and $B$ be points of a bounded connected domain $D$ in $E_{n}$ such that $M$ is a subset of $D$ and no set $M_{n}$ separates $A$ from $B$ in $D$. Then $D-M$ contains an arc from $A$ to $B$. (3) If $M$ lies in $E_{2}$, no $M_{n}$ separates $E_{2}, K$ is a component of a set $M_{n}$, and $e$ is any positive number, there exists a simple closed curve $J$ such that every point of $J$ is at a distance from $K$ less then $e$ and such that $J$ and $M$ have no point in common.

23. Mr. L. W. Cohen: On sets homeomorphic with linear sets.

Definitions of closed arcs, half open arcs, and open arcs are stated for a separable metric space, and such sets are shown to be homeomorphic respectively with closed, half open, and open intervals on the linear continuum. The following theorem is proved: Necessary and sufficient conditions that a set $M$ in a separable metric space be homeomorphic with a linear set are that (1) the components of $M$ are points of arcs; (2) if $p$ is a point component of $M$, then $\operatorname{dim}_{p} M=0$; (3) if $a$ is an end point of an arc component $T$ in $M$, then $\operatorname{dim}_{a}(M-T+a)=0 ;(4)$ if $p$ is a point of an arc component $T$ distinct from its end points, then $p$ is not a limit point of $M-T$. In this theorem an arc is either closed, half open, or open. 


\section{Dr. P. A. Smith: The regular components of a surface transformation.}

Regular components are maximal connected regions of "uniform approach" toward certain invariant limit sets on the surface. These components have been studied by Birkhoff and Smith in a paper to appear in the Journal de Mathématiques, but only on surfaces of genus zero. The results are here extended to the general case.

25. Professor E. W. Chittenden: On covering theorems in general topological space.

A family $F$ of sets $G$ of elements of a class $P$ is said to be a covering of a subset $E$ of $P$ and to be of type $T$ in case each element $p$ of the set $E$ belongs to a set $G$ of $F$ and is also in the relation $T$ to some set $G$ of $F$. The undefined relation $T$ is assumed to be definite between elements and subsets of $P$. For example, in a topological space the relation $T$ may be defined to mean that the element $p$ is interior to $G$. A covering $F$ is called reducible provided it contains a covering of the same type and of lower cardinal number. The covering theorems of Borel, Lebesgue, and Lindelöf, together with certain extensions suggested by Alexandroff, Urysohn, and Hildebrandt, are considered in relation to this concept of reducibility. In particular, the paper contains sets of necessary and sufficient conditions for the reducibility of every covering $F$ (of a set $E$ ) which is of type $T$. The results obtained are applied to the theory of topological spaces in general.

\section{Dr. P. A. Smith: Transformations of the boundary of a simply connected invariant region.}

This paper is a report on certain questions which arise in a topological study of the structure of surface transformations.

\section{Professor Henry Blumberg: On various metric pro- perties of general sets and functions.}

In this paper the following theorems are proved: If $S$ is a point set in euclidean $n$-space, it may be uniquely represented (sets of measure zero regarded as negligible) in the form $S=M+N$, where $M$ is measurable and $N$ is such that at every point of $N$ the exterior metric density of $N$ and also that of the complement of $N$ is equal to 1 . If $A$ and $B$ are any two sets whatever, overlapping or not, the exterior metric density of $A$ is 0 or 1 at every point of $B$, with the possible exception of a set of measure zero. If $f(x)=f\left(x_{1}, \cdots, x_{n}\right)$ is an arbitrary real function and $u_{m}(x), l_{m}(x)$ represent the upper and lower metric limits of $f$ at $x$, that is, the largest and smallest values of $\eta$ for which $(x, \eta)=\left(x_{1}, x_{2}, \cdots, x_{n}, \eta\right)$ is "approached" but not "infinitesimally approached," in the metrical sense, by the surface $y=f(x)$, then $u_{m}(x), l_{m}(x)$ are "metrically upper-semi-continuous," and, moreover, approximately continuous, in the sense of Denjoy, almost everywhere; hence $u_{m}(x)$ and $l_{m}(x)$ are measurable. Therefore, given any function $f(x)$, there exist two measurable functions $m_{1}(x)$ and $m_{2}(x)$ such that 
$m_{1}(x) \geqq f(x) \geqq m_{2}(x)$ almost everywhere, and the metrical approach of $y=f(x)$ at $\left(x, m_{1}(x)\right)$ as well as at $\left(x, m_{2}(x)\right)$ is "full," in the sense of metric density.

28. Professor Henry Blumberg: Note concerning the inductive principle.

It seems not to have been remarked that we may formulate the inductive principle for all linearly ordered sets as follows, and thereby comprehend in our formulation the particular applications to the normally ordered sets and to the linear continuum: If the property $P$ holds for all the elements of some segment $S$ of a linearly ordered set $A$, and if the validity of $P$ for all the elements of a segment $S$ of $A$ implies its validity for all the elements of some proper supersegment $S^{\prime}$ of $A$, then $P$ holds for all the elements of $A$. The principle is extensible to multiply ordered sets.

29. Professor H. M. Gehman: Concerning certain types of non-cut points, with an application to continuous curves.

A point $P$ of a continuum $M$ is called a point of type 1 , if given any positive number $\epsilon$, there exists an $M$-domain containing $P$ of diameter less than $\epsilon$ whose complement is connected. Similarly, $P$ is a point of type 2, if there exists an open set in $M$ containing $P$ of diameter less than $\epsilon$ whose complement is connected. A point of type 1 is a point of type 2, and a point of type 2 is a cut point, but not conversely, in either case. Certain properties of points of types 1 and 2 are proved in this paper, and an application is found in the proof of the following theorem: If $M$ is a continuous curve (bounded or unbounded) in space of any finite number of dimensions, and if $P$ is a non-cut point of $M$, then given any positive number $\epsilon$, there exists an $M$-domain containing $P$ of diameter less than $\epsilon$ whose complement is connected; if $P$ is a cut point of $M$, then given any positive number $\epsilon$, there exists an $M$-domain containing $P$ of diameter less than $\epsilon$ whose complement consists of a finite number of maximal connected sets.

30. Professor G. T. Whyburn: Concerning certain accessible points of plane continua.

Let $K$ denote the set of all those points of a plane continuum $M$ each of which is accessible from at least two complementary domains of $M$. Then (1) if $H$ is any uncountable subset of $K$, there exist points $X$ and $Y$ of $H$, an uncountable subset $E$ of $H$, complementary domains $R_{1}$ and $R_{2}$ of $M$, and two continua $L$ and $N$ such that (a) $L \cdot N=X+Y$, (b) $L+N=M$, (c) every point of $E$ is a cut point of $N$ and is accessible from both $R_{1}$ and $R_{2}$; (2) all, save possibly a countable number, of the points of $K$ are points of Menger order two of $M$; (3) if $G$ is the collection of all the finite subsets of $M$ each of which is an irreducible cutting of $M$, and $T$ is the sum of all the point sets of the collection $G$, then all, save possibly a countable number, of the points of $T$ are points of Menger order two of $M$. An interesting generalization of (1) is given which can be applied in the study of the relation between a subcontinuum of a plane continuous curve and its complement in that curve. 


\section{Professor G. T. Whyburn: A generalized notion of ac-} cessibility.

A continuum $K$ will be said to be accessible from a point set $R$ provided that if $A$ is any point of $R$, a bounded continuum exists which contains both $A$ and $K$ and is a subset of $R+K$. The following results are established. (1) If $R_{1}, R_{2}$, and $R_{3}$ are mutually exclusive connected point sets in a plane $S$, there do not exist, in $S-\left(R_{1}+R_{2}+R_{3}\right)$, three mutually exclusive continua each of whch is accessible from both $R_{1}$ and $R_{2}$ and contains a limit point of $R_{3}$. (2) Every component of the $M$-boundary of every connected open subset $R$ of a continuous curve $M$ (in $n$ dimensions) is regularly accessible from $R$. (3) For open subsets of a continuous curve, accessibility by continua as above defined is equivalent to accessibility by a special kind of a continuum, which is an "ideal arc" all of whose elements are points except possibly one. (4) The following properties are equivalent for continua $M$ (in $n$ dimensions): ( $\alpha$ ) every subcontinuum of $M$ is a continuous curve, $(\beta)$ every connected open subset of $M$ has property $S$, and $(\gamma)$ every point of the $M$-boundary of every connected open subset $R$ of $M$ is regularly accessible from $R$.

32. Mr. P. M. Swingle: A certain type of continuous curve and related point sets.

This paper is a study of continuous curves every subcontinuum of which is itself a continuous curve; special sections are devoted to (1) continua irreducible between two points, and (2) sets which are composed of the points in sets of arcs joining two distinct closed sets, with a view to determining the relation between the number of possible distinct arcs and the number of common points. The following conditions are each shown to be necessary and sufficient that every subcontinuum of a continuous curve be itself a continuous curve: (1) If $P, A$ and $B$ are any three points of a subcontinuum $N$ of $M$ such that there exists no point, distinct from $P$, contained in every arc of $N$ from $P$ to $A+B$, then there is an $\operatorname{arc} A P B$ in $N$. (2) If $N$ is a connected subset of $M, A$ and $B$ are distinct points of $N, L$ is the set consisting of the points of $\operatorname{arcs} A B$ of $N^{\prime}$, and $Q=L \times N$, then $Q$ is connected. (3) If $L$ is the set consisting of points of a set of arcs of $M$ joining two distinct closed sets $E$ and $F$, then every point of $L^{\prime}$ is contained in an arc of $L^{\prime}$ joining $E$ and $F$. It is also shown that in order that every subcontinuum of a continuum $M$ be a continuous curve it is sufficient that if $A$ and $B$ are any two distinct points of $M$ there exist a finite number of points which separate $A$ and $B$ in $M$.

33. Mr. E. W. Miller: Note on the relation between component and quasi-component in a closed point set.

In a metric space, if $M$ is a compact and closed set and $P$ is a point of $M$, then the quasi-component of $M$ determined by $P$ and the component of $M$ determined by $P$ are identical. If the condition of compactness is removed this is no longer true. However, in a metric space every bounded subset of which is compact, it is shown that if $M$ is a closed set then in 
order that the quasi-component of $M$ determined by $P$ should be identical with the component of $M$ determined by $P$ it is sufficient that the component be compact.

\section{Dr. W. L. Ayres (National Research Fellow): On con- tinua which are disconnected by the omission of any point.}

There exists a plane continuum $M$ such that (1) every point of $M$ is a cut point of $M,(2)$ every continuum $K$ such that every point of $K$ is a cut point of $K$ is homeomorphic with some subset of $M$. In order that every point of a plane continuum $M$ be a cut point of $M$ either of the following conditions is necessary and sufficient: (1) every point of $M$ be accessible from two unbounded complementary domains, (2) $M$ be non-dense, the boundary of every complementary domain be an open curve and only a finite number of complementary domains have points within any circle. If $P$ is a non-cut point of a continuous curve $M$ in $n$ dimensions and $\epsilon$ is any positive number, there exists a continuous curve $N$ and a positive number $\delta$ such that (1) $M$ contains $N$, (2) the diameter of $N$ is less than $\epsilon$, (3) every point of $M$ whose distance from $P$ is less than $\delta$ belongs to $N$, (4) $M-N$ is connected.

35. Dr. T. W. Moore (National Research Fellow): Extended results in elimination.

This paper presents a means of elimination for double binary forms in sets of digredient variables. The fundamental problem is to get the condition that three such forms have a common solution expressed as a determinant involving no extraneous factors and not vanishing identically. The method employed is an extension of that used by Morley and Coble for forms in one set of variables published in their article New results in elimination, in the American Journal of Mathematics, vol. 49 (1927), No. 4. A covariant containing the coefficients of all three forms and vanishing when they have a common solution is used to obtain this determinant representation. The method has already been applied to a limited range of cases and appears capable of extension to forms containing other than binary variables.

\section{Dr. T. W. Moore: On the complete system of combinants of two binary quintics.}

This paper contains a system of combinants of two binary quintic forms, derived as the simultaneous system of covariants of their first and third transvectants, an octavic and a quartic respectively. It is a continuation of a paper presented to this Society in February, 1927 (see this Bulletin, vol. 33, p. 260) in which the case of the invariant combinants only was considered. The list of forms found in the system contains 113 members. All these are equivalent to the 69 independent forms in the complete system of the binary octavic apolar to the two given quintics, but the exact connection has not yet been established. 


\section{Professor J. R. Musselman: A planar imprimitive} group of order 216.

In a recent paper in the American Journal, the existence of a planar imprimitive group of order 216 connected with four Hesse configurations was shown by the writer. The purpose of the present paper is to discuss the group and its associated geometry in detail. Of special interest is a configuration of twelve triangles. A new canonical form used in discussing four-fold projective triangles leads to a ruler construction for them and a compass construction for a Clebsch six-point. The group in $S_{n}$ is of order $6^{n}(n+1) !$.

38. Professor Oystein Ore: Arithmetical theory of Galois fields. Second communication.

In the author's first communication on this subject it was shown that the complete system of residues for prime-ideal powers can be derived by successive adjunctions of roots of binomial and trinomial congruences, corresponding to the different subgroups of the "Zerlegungs"-group of Hilbert. In the present paper various consequences of these results are derived, and it is proved that the normal congruences are either all binomial or all trinomial. It then follows that in the binomial cases both the "Trägheits"-group and the "Verzweigungs"-group of Hilbert must be cyclic groups.

39. Dr. Raymond Garver: A new normal form for quartic equations.

This paper has appeared in the May-June issue of this Bulletin.

40. Dr. Raymond Garver: The Tschirnhaus transformation.

In this paper, which is to appear in the Annals of Mathematics, the author makes what he thinks is the first attempt to collect and consider the various results that have been obtained by the use of the Tschirnhaus transformation. A bibliography of 100 titles accompanies the paper.

41. Professor F. D. Murnaghan: The stress components in an elastic solid subjected to a finite deformation.

When an elastic solid is subjected to a finite strain it is shown that the stress components are derivable from the elastic energy $\phi$, which is assumed to be a function of the six strain components $\left(e_{1}, \cdots, e_{6}\right)$, by means of the equations $X_{x}=\partial \phi / \partial e_{1}-\left(2 e_{1} \partial \phi / \partial e_{1}+e_{5} \partial \phi / \partial e_{5}+e_{6} \partial \phi / \partial e_{6}\right) ; \quad Y_{z}=\frac{1}{2} \partial \phi / \partial e_{4}$ $-\left(2 e_{4} \partial \phi / \partial e_{2}+e_{3} \partial \phi / \partial e_{4}+e_{5} \partial \phi / \partial e_{6}\right) ; \quad Z_{y}=\frac{1}{2} \partial \phi / \partial e_{4}-\left(2 e_{4} \partial \phi / \partial e_{3}+e_{2} \partial \phi / \partial e_{4}\right.$ $\left.+e_{6} \partial \phi / \partial e_{5}\right)$. In these expressions $e_{1}=\partial U / \partial x-\frac{1}{2}\left[(\partial U / \partial x)^{2}+(\partial V / \partial x)^{2}\right.$ $\left.+(\partial W / \partial x)^{2}\right] ; \quad 2 e_{4}=\partial W / \partial y+\partial V / \partial z-[(\partial U / \partial y)(\partial U / \partial z)+(\partial V / \partial y)(\partial V / \partial z)$ $+(\partial W / \partial y)(\partial W / \partial z)]$, etc., where $(U, V, W)$ is the displacement vector of any particle of the medium. If we assume that the stress tensor is symmetric, we have three equations of the type $Y_{z}=Z_{y}$, which form a complete 
system, and $\phi$ must be a function of the three strain invariants. Hence for a non-isotropic, that is, crystalline medium, we must give up the assumption that the energy of deformation is a function of the strain components.

42. Professor F. D. Murnaghan and Mr. Carl Kaplan: On the foundations of electromagnetic theory. Preliminary communication.

An examination is made of the connection between the two fundamental covariant alternating tensors of rank two, namely the $(B, c E)$ and the $(D,-c H)$ tensors, which appear in Maxwell's equations. It appears that the usual equations $B=\mu H$ and $D=\epsilon E$ are but special cases of a more general theory which would put $B=\alpha D+\beta H, E=\gamma D+\delta H$, where $\alpha, \beta, \gamma, \delta$ are constants.

43. Dr. Hillel Poritsky (National Research Fellow): ndimensional potential theory.

In this paper we indicate the elements of the theory of $p$-vector potentials in $n$-dimensional euclidean space, and generalize to $n$ dimensions some of well known propositions of three-dimensional potential theory. As an example, we obtain one chain of theorems that reduces for $n=3$ to three links of which the outer ones are equivalent to Gauss's flux theorem while the middle one is equivalent to the linkage theorem of electromagnetism: the work done in carrying a unit magnetic pole around a closed path $C$ in the field of unit electric current flowing in a circuit $\gamma$ is equal to $4 \pi n$, where $n$ is the number of times $C$ links with $\gamma$.

\title{
44. Professor W. A. Hurwitz: On Bell's arithmetic of Boo- lean algebra.
}

This paper suggests a modification of the arithmetic of Boolean algebra given by Bell (Transactions of this Society, vol. 29, pp. 597-611) by proposing a definition of congruence which retains all the properties postulated by Bell and also restores several analogies to rational arithmetic.

\author{
Arnold Dresden, \\ Associate Secretary.
}

